\title{
Non-computability, unpredictability, and financial markets
}

\author{
Daniel S. Graça \\ CEDMES/FCT da Universidade do Algarve, Portugal \& \\ SQIG - Instituto de Telecomunicações, Portugal
}

January 10, 2012

\begin{abstract}
One of the most significant achievements from theoretical computer science was to show that there are non-computable problems, which cannot be solved through algorithms. Although the formulation of such problems is mathematical, they often can be interpreted as problems derived from other fields, like physics or computer science. However no noncomputable problem with economical or financial inspiration has been presented before. Here we study the problem of valuation: given some adequate data, find the value of an asset. Valuation is modeled mathematically by the discounted cash flow operator. We show, using surprisingly simple arguments, that this operator is not computable. Since, theoretically, financial markets should trade assets based on their fair value, our result suggests that unpredictability of such markets may partially stem from inherent non-computable behavior. A discussion of this result is also included.
\end{abstract}

\section{Introduction}

It is well-known that there are non-computable problems which cannot be solved by algorithms. Turing [1] presented the most famous non-computable problem, the Halting Problem, but soon after many mathematical problems were shown to be non-computable, e.g. Hilbert's 10th problem [2].

Non-computability is tightly related to the issue of unpredictability. A noncomputable problem may be completely deterministic in the sense that given an input, there is only one possible mathematical solution. Yet, because of the problem's non-computable nature, its solution cannot in general be obtained by any algorithm, computer program, mathematical formula, rule of thumb, etc. Thus, in a very strong sense, one may say that non-computable problems are unpredictable.

Many non-computable problems originate from applications. But no problem inspired from finance or economics has been previously shown to be noncomputable. In this paper we tackle a problem from finance and show that it is 
non-computable. We consider the following question (the problem of valuation): given an asset (a stock, an house to be rented, etc.) can we compute its fair value from some suitable input data?

Our motivation for asking this kind of question comes from stock market charts. If one sees the variation of a stock price within one single day, its exact evolution seems rather unpredictable. Several explanations have been proposed for this behavior: markets may be speculative, irrational, and most useful information to markets is unknown. Certainly all these factors, which do not appear on the ideal world of mathematical models, account for unpredictability on the real world. But is unpredictability only a defect from perhaps excessive modeling or does it go deeper than that? Can unpredictability be inherent to the mathematical models of finance? According to financial theory, the fair (or fundamental) value of an asset can be determined using the Discounted Cash Flow Model (DCFM), which we will analyze in more detail in Section 2. The DCFM is completely deterministic: given complete knowledge about the data of an asset (includes information about the future) we can mathematically determine the fundamental value of the asset.

But determinism does not imply predictability. Examples abound in chaos theory (recall the famous butterfly effect) and even in theoretical computer science, with non-computable problems.

Here we will show that, while the DCFM is completely deterministic, the DCFM is non-computable and therefore intrinsically unpredictable. By other words, even if we have exact knowledge of the inputs for the DCFM (in practice this information is hard - if not impossible - to obtain, since it relies on the future) we cannot, in general, compute the fair price of an asset under the DCFM (although this can be done for some particular cases. The point is that it cannot be done "in general").

The aim of this paper is not to introduce sophisticated mathematical machinery, but rather to show that results from theoretical computer science can shed new light on the hardness of problems from fields like finance or economics.

In Section 2 we recall the DCFM model. Section 3 recalls material from computability with real numbers (Computable analysis). Section 4 introduces the main result of this paper: non-computability of the DCFM operator. In Section 5 we analyze the result in more detail.

\section{The Discounted Cash Flow Model}

The discounted cash flow model (DCFM) is a theoretical model which aims to solve the valuation problem. It is consider to be the foundation on which all other approaches to pricing are built (see e.g. [3], [4]), although, for practical reasons, the majority of valuations is done by comparing the value of similar assets (relative valuation - see e.g. [3]).

The DCFM says that an asset (a stock, an house to be rented, a bond - debt issued by a government or company, etc.) is worth the discounted sum of all of its future earnings. Usually this is what is mean as the fair (or fundamental) 
value of the asset. In the discounted cash flow model, one takes two inputs, the discount rate (see below for details) and the future earnings of the asset, and get as a result the present value of the asset. Therefore this model defines a function $f$ which maps the input (discount rate, future earnings) to some element of $\mathbb{R}$ (the value of the asset).

Let us describe what is the discount rate. Suppose that you have a claim over some company ABC which guarantees that it will pay you $\$ 100$ exactly within one year. However, you need money urgently and want to sell this claim now. A potential buyer must ask itself the following: how much next year's $\$ 100$ is worth today? Suppose that the buyer could put his money into a savings certificate yielding an interest rate of $r=5 \%$ with no risk (the risk-free interest rate). Then, for the buyer, these next year's $\$ 100$ are not worth more than

$$
\frac{100}{(1+r)}
$$

or approximately $\$ 95.24$ today. If this claim over the company is as safe as the savings certificate (i.e. no risk), then one can say that next year's $\$ 100$ are worth (1) with $r=5 \%$. However, claims over companies are not risk-free. Although some companies are safer than others, none can guarantee no risk. It is usually assumed (cf. [4]) that investors are risk-averse, meaning that all other things being equal, an investor will prefer the less risky investment, in this case the savings certificate. Therefore, in order to buy our claim over company ABC, an investor will ask a risk premium, let's say $r=10 \%$ in (1) for a relatively safe blue-chip company, or $r=30 \%$ for an even riskier investment like a claim over a start-up company. The value $r$ is called the discount rate.

This discount rate is one of the inputs to the function $f$ defined above. It can be determined, for example, by using the well-known Capital Asset Pricing Model (CAPM). The interested reader can find further details in [4].

Under the DCFM, the value of an asset which pays $a$ dollars each year for $N$ years, with discount rate $r$, and in the end of these $N$ years returns $b$ dollars is

$$
\frac{b}{(1+r)^{N}}+\sum_{t=1}^{N} \frac{a}{(1+r)^{t}}
$$

In general, if the asset is never sold, its value will be

$$
\sum_{t=1}^{\infty} \frac{a}{(1+r)^{t}}
$$

Of course we have made, as usual (see e.g. [3], [4]), some assumptions which may not be true: we assumed that earnings as well as the discount rates remain constant over time. This does not happen in reality: a company is expected to have (hopefully) growing earnings and its discount rate may change over time (e.g. because the risk-free interest rate changes and/or because its risk profile may change: a young and risky start-up may mature into a solid blue-chip 
company). For this reason we can say that the value of an asset is

$$
\sum_{t=1}^{\infty} e(t) u(t)
$$

where $e(t)$ is the earnings paid by the asset at time $t$ and $u$ is the discount function (in the preceding examples we used the simplistic assumption that $e(t)=a$ and $u(t)=\frac{1}{(1+r)^{t}}$ for all $\left.t \in \mathbb{N}\right)$.

This can be defined formally as follows.

Definition 1 Let $\mathcal{A}$ be some financial asset. Its earnings function is a function $e: \mathbb{N} \rightarrow \mathbb{R}$ and its discount function is a function $u: \mathbb{N} \rightarrow \mathbb{R}$. The value of the asset under the DCFM is

$$
\sum_{t=1}^{\infty} e(t) u(t)
$$

Definition 2 The DCFM operator is a function $f: \mathbb{R}^{\mathbb{N}} \times \mathbb{R}^{\mathbb{N}} \rightarrow \mathbb{R}$ such that for any financial asset with earnings function $e: \mathbb{N} \rightarrow \mathbb{R}$ and discount function $u: \mathbb{N} \rightarrow \mathbb{R}, f(e, u)$ returns the value of the asset under the DCFM. More, one has

$$
f(e, u)=\sum_{t=1}^{\infty} e(t) u(t)
$$

\section{Computable Analysis}

In this paper we will be computing with real numbers. To achieve this objective, we introduce some definitions and results from Computable Analysis. Books on the subject are, for example, [5], [6], [7] though we recommend the excellent tutorial [8]. We also suppose that the reader is familiar with Turing machines and classical computability theory ([9] is a good reference on the subject).

Turing, in its seminal paper [1] where he introduced Turing machines, was already interested in the subject of computing with real numbers. He proposed to code each real as its decimal expansion over some tape and then to make computations over this symbolic representation.

This is the idea behind Type-2 machines (see e.g. [5]). These machines are like Turing machines, with a few exceptions: (i) there is a read-only input tape, where the input is written. Contrarily to Turing machines, an infinite number of cells may have non-blank symbols; (ii) there is a write-only output tape, where the output is written. The head in this tape cannot move left. This guarantees that at any given time a partial result is in the output tape. For instance, after 1000 steps of computation, we could have written in the output tape "3.1415". Since the head cannot move left, we cannot alter this partial result, but just add decimal digits i.e. precision to the result. In principle one will need an infinite amount of time to get the exact result, but the important point is that, at any time, we have a partial result with improving accuracy over time. 
Actually, as noted by Turing in the correction [10] of its original paper [1], the decimal expansion is not good for computing with real numbers. This is because decimal expansion does not preserve the topology of the real line (e.g. " $0.999 \ldots$..." and " $1.00 \ldots$.." are far away as sequences of symbols, yet they represent the same real number). For instance consider the function $f: \mathbb{R} \rightarrow \mathbb{R}$ defined by $f(x)=3 x$. It is expectable that this simple function would be computable. But, using decimal representation of real numbers, it is not [5].

For this reason different representations, which respect the topology of the real line, are used to encode real numbers as sequences of symbols, for example by means of Cauchy sequences, sequences of rational intervals converging to the real number, etc. The "typical" representations used are equivalent in terms of computability (see [5]). Other notions of computability over the real numbers which do not involve Type-2 machines can be presented (see e.g. [6] or [7]), yet they can be shown to be equivalent to this approach [5].

The representation of real numbers that we will use is the following.

Definition 3 A sequence $\left\{r_{n}\right\}_{n \in \mathbb{N}}$ of rational numbers is called a $\rho$-name of a real number $x$ if there exist three functions $a, b, c$ from $\mathbb{N}$ to $\mathbb{N}$, such that for all $n \in \mathbb{N}, r_{n}=(-1)^{a(n)} \frac{b(n)}{c(n)+1}$ and

$$
\left|r_{n}-x\right| \leq \frac{1}{2^{n}}
$$

We can also give the notion of $\rho$-names for sequences of real numbers.

Definition 4 A double sequence $\left\{r_{l, k}\right\}_{l, k \in \mathbb{N}}$ of rational numbers is called a $\rho$ name for a sequence $\left\{x_{l}\right\}_{l \in \mathbb{N}}$ of real numbers if there are three functions $a, b, c$ from $\mathbb{N}^{2}$ to $\mathbb{N}$ such that, for all $k, l \in \mathbb{N}, r_{l, k}=(-1)^{a(l, k)} \frac{b(l, k)}{c(l, k)+1}$ and

$$
\left|r_{l, k}-x_{l}\right| \leq \frac{1}{2^{k}}
$$

Notice that a sequence of real numbers may be interpreted as a function $f: \mathbb{N} \rightarrow \mathbb{R}$, where $f(l)=x_{l}$. Thus the earnings function and the discount rate function of Section 2 can be described by $\rho$-names for their respective sequence. This characterization of sequences will be used in Section 4 .

Definition 5 A real number $x$ (a sequence $x=\left\{x_{l}\right\}_{l \in \mathbb{N}}$ of real numbers) is called computable if there is a Type-2 machine that, without using any input, outputs a $\rho$-name for $x$.

Examples of computable real numbers include: integers, rational numbers, algebraic numbers $\left(\sqrt{2}, 5^{\frac{2}{7}}\right.$, etc.), $\pi$, e, etc. The set of computable numbers is closed under many operations: addition, multiplication, division, etc (see e.g. [5], [6]).

Very few non-computable numbers are explicitly known, although "almost all" real numbers are not computable (the set of computable numbers is countable, while the set of real numbers is not. This implies that "almost all" real 
numbers are not computable). To the knowledge of the author, the only noncomputable numbers explicitly presented in the literature are those which result from the following Proposition, from Section 0.2 of [7].

Proposition 6 Let $a: \mathbb{N} \rightarrow \mathbb{N}$ be a one to one computable function generating a recursively enumerable nonrecursive set. Then the series

$$
\sum_{i=0}^{\infty} 2^{-a(i)}
$$

converges to a non-computable real.

Notice that functions $a: \mathbb{N} \rightarrow \mathbb{N}$ in the conditions of the previous proposition do exist (actually there are infinitely many), since there are nonrecursive sets (this can be shown through a diagonalization argument) which can be enumerated, see e.g. [11]. Let us analyze the meaning of

$$
x=\sum_{i=0}^{\infty} 2^{-a(i)}
$$

being not computable. First we notice that this number is "semi-computable": one can compute a sequence of rational numbers $r_{k}$ which converge to $x$. To see this it is enough to take $r_{k}=\sum_{i=0}^{k} 2^{-a(i)}$. What is missing to have computability as in Definition 3 is the bound $1 / 2^{n}$ in (2). By other words, we can compute an approximation $r_{k}$ to the real number $x$ and we know that $r_{k} \rightarrow x$. But if we use $r_{k}$ as an approximation of $x$ we cannot (algorithmically) know how far $r_{k}$ is from $x$.

By other words, if $x$ is a non-computable real number, there is no algorithm which solves the following problem: "Given as input $n \in \mathbb{N}$, output as result a rational number $r$ such that $|r-x| \leq 2^{-n}$ ". This means that we cannot compute an approximation of $x$ within a given accuracy.

For completeness, and because this result is central for our paper, we finish this section with the proof of Proposition 6, adapted from [7]. In a first reading, the reader may want to skip this proof.

Lemma 7 Let $a: \mathbb{N} \rightarrow \mathbb{N}$ be a one to one computable function generating a recursively enumerable nonrecursive set $A$. Define the function $w: \mathbb{N} \rightarrow \mathbb{N}$ by

$$
w(n)=\max \{m: a(m) \leq n\} .
$$

Then there is no computable function $f: \mathbb{N} \rightarrow \mathbb{N}$ such that $f(n) \geq w(n)$ for all $n \in \mathbb{N}$.

Proof. Suppose, by absurd, that there is a computable function $f: \mathbb{N} \rightarrow \mathbb{N}$ such that $f(n) \geq w(n)$ for all $n \in \mathbb{N}$. Then the following procedure decides the set $A$ :

(i) Given input $n$, compute $f(n)$ 
(ii) For each $m \leq f(n)$, check whether $a(m)=n$. If $a(m)=n$ for some $m \leq f(n)$, then $n \in A$. If $a(m) \neq n$ for all $m$, from (3), then it must be $n \notin A$. Since the previous algorithm decides $A$, this set must be recursive, which is absurd since $A$ is nonrecursive by hypothesis. Therefore there is no computable function $f: \mathbb{N} \rightarrow \mathbb{N}$ such that $f(n) \geq w(n)$ for all $n \in \mathbb{N}$.

Lemma 8 Let $a: \mathbb{N} \rightarrow \mathbb{N}$ and $w: \mathbb{N} \rightarrow \mathbb{N}$ be defined as in the preceding lemma. Take

$$
s_{k}=\sum_{m=0}^{k} 2^{-a(m)}
$$

and let $x=\lim _{k \rightarrow+\infty} s_{k}$ (this limit exists since $a$ is one to one). Define the "optimal modulus of convergence" $e^{*}(n)$ to be the smallest integer such that

$$
k \geq e^{*}(n) \quad \text { implies } \quad\left|x-s_{k}\right| \leq 2^{-n} .
$$

Then $w(n)=e^{*}(n)$.

Proof. First we show that it must be $e^{*}(n) \geq w(n)$. By definition of $w$ we have that

$$
a(w(n)) \leq n .
$$

Suppose, by absurd, that $k=e^{*}(n)<w(n)$. Then the series

$$
x-s_{k}=\sum_{m=k+1}^{+\infty} 2^{-a(m)}
$$

includes the term $2^{-a(w(n))}$. Since all terms in the right-hand side of $(7)$ are positive and $2^{-a(w(n))} \geq 2^{-n}$ by (6), we conclude that $\left|x-s_{k}\right|>2^{-n}$, which contradicts (5) since $k=e^{*}(n)$. Therefore it must be $e^{*}(n) \geq w(n)$. We now show that

$$
k \geq w(n) \quad \text { implies } \quad\left|x-s_{k}\right| \leq 2^{-n}
$$

which shows, in conjunction with the fact that $e^{*}(n) \geq w(n)$, that $e^{*}(n)=w(n)$. Suppose that $k \geq w(n)$. Then (7) contains no terms $2^{-a(m)}$ satisfying $a(m) \leq n$. Therefore

$$
\left|x-s_{k}\right|=\sum_{m=k+1}^{+\infty} 2^{-a(m)} \leq \sum_{i=n+1}^{+\infty} 2^{-i}=2^{-n}
$$

which proves the result.

Lemma 9 Let $a: \mathbb{N} \rightarrow \mathbb{N}$ be a one to one computable function generating a recursively enumerable nonrecursive set and let $\left\{s_{k}\right\}_{k \in \mathbb{N}}$ be given by (4) and let

$$
x=\lim _{k \rightarrow+\infty} s_{k}=\sum_{m=0}^{+\infty} 2^{-a(m)} .
$$

Then there is no computable function $e: \mathbb{N} \rightarrow \mathbb{N}$ satisfying

$$
k \geq e(n) \quad \text { implies } \quad\left|x-s_{k}\right| \leq 2^{-n} \text { for all } n \in \mathbb{N} .
$$


Proof. Suppose, by absurd, that there is a computable function $e$ satisfying (9). Then, from the preceding lemma, it must be $e(n) \geq w(n)$ for all $n \in \mathbb{N}$. But, by Lemma 7, e cannot be computable, which is an absurd.

In the lemma below we use the following notion: $\left\{s_{n}\right\}_{n \in \mathbb{N}}$ is a computable sequence of rational numbers if

$$
s_{n}=(-1)^{a(n)} \frac{b(n)}{c(n)+1}
$$

for all $n \in \mathbb{N}$, where $a, b, c$ are computable functions from $\mathbb{N}$ to itself.

Lemma 10 Let $\left\{s_{n}\right\}_{n \in \mathbb{N}}$ be a computable sequence of rational numbers which converges monotonically upwards to a limit $x$, i.e. $s_{0} \leq s_{1} \leq \ldots \leq s_{n} \leq \ldots \leq x$. Then the number $x$ is computable iff there is a computable function e such that (9) holds.

Proof. Suppose that there is a computable function $e$ satisfying (9). Then $\left|r_{n}-x\right| \leq 2^{-n}$ where

$$
r_{n}=(-1)^{a(e(n))} \frac{b(e(n))}{c(e(n))+1}
$$

and $a, b, c$ are given by (10), i.e. $\left\{r_{n}\right\}_{n \in \mathbb{N}}$ is a computable $\rho$-name for $x$ and therefore $x$ is computable.

Reciprocally, suppose that $x$ is computable. Then $x$ admits a computable $\rho$-name $\left\{r_{n}\right\}_{n \in \mathbb{N}}$ satisfying $\left|r_{n}-x\right| \leq 2^{-n}$. Then define

$$
e(n)=\min \left\{k \in \mathbb{N}: s_{k} \geq r_{n}\right\} .
$$

It is easy so see, since $\left\{s_{n}\right\}_{n \in \mathbb{N}}$ is computable and converges monotonically to $x$, and $\left\{r_{n}\right\}_{n \in \mathbb{N}}$ is computable, that $e: \mathbb{N} \rightarrow \mathbb{N}$ is a computable function and satisfies (9).

We now prove Proposition 6.

Proof of Proposition 6. It is easy to see that $\left\{s_{n}\right\}_{n \in \mathbb{N}}$, where $s_{n}$ is defined by (4) yields a computable sequence of rational numbers which converge monotonically upwards to (8). By Lemma 9, no computable function $e$ exists satisfying (9). Thus, by Lemma 10, (8) cannot be a computable real.

\section{Presentation of results}

The following result shows that the DCFM operator is not computable.

Lemma 11 There is a computable earnings function e and a computable discount rate function $u$ such that $f(e, u)$ is not computable, where $f: \mathbb{R}^{\mathbb{N}} \times \mathbb{R}^{\mathbb{N}} \rightarrow \mathbb{R}$ is the DCFM operator.

Proof. Take, for instance, $e(n)=1000$ for all $n \in \mathbb{N}$ (1000 is an arbitrary number. Any computable number could be used) and

$$
u(n)=\frac{1}{2^{a(n)}}
$$


where $a: \mathbb{N} \rightarrow \mathbb{N}$ is a one to one computable function generating a recursively enumerable nonrecursive set. Notice that both functions $e$ and $u$ are computable. Thus

$$
f(e, u)=\sum_{t=1}^{\infty} e(t) u(t)=1000 \sum_{t=1}^{\infty} \frac{1}{2^{a(n)}},
$$

but the value $f(e, u)$ is not computable (if, by absurd, $f(e, u)$ was computable, then so would be $f(e, u) / 1000$ since the set of computable numbers is closed under division. But then the value $\sum_{t=1}^{\infty} 2^{-a(i)}$ would be computable, a contradiction to Proposition 6).

The previous lemma implies the following result.

Theorem 12 Let $f: \mathbb{R}^{\mathbb{N}} \times \mathbb{R}^{\mathbb{N}} \rightarrow \mathbb{R}$ be the DCFM operator. Then $f$ is not computable.

Proof. If $f$ would be computable, then by a standard result from Computable Analysis (see e.g. [8]), the value $f(e, u)$ should be computable if both $e$ and $u$ are computable. But this is a contradiction to the previous lemma.

\section{Criticism}

In the previous section we have seen that the DCFM operator is non-computable. Here we analyze this result more carefully to understand if non-computability is genuine or if it follows from using pathological assumptions.

1. A first criticism is the use of an infinite sum in the right-hand side of (6). It might seem that this series is an artifact of modeling since no one will live forever and thus one would not be interested in earnings that an asset might yield beyond his/her death. Actually this problem has more to do with the DCFM than with our result, but let us analyze it. We begin by mentioning that there are classes of assets that ideally satisfy $e(t)>0$ for all $t \in \mathbb{N}$. For example, the United Kingdom government has issued in the past bonds, called consols, which make fixed periodic payments that continue indefinitely in time [4]. These consols are actively traded in the bond market and, according to the DCFM, a series must be used to determine their value. A buyer will value a consol based on the earnings it will provide until sold, plus the sell value. But the sell value depends on the earnings the next buyer will get. Continuing this reasoning, it is not difficult to see that the initial value of the consol for the first holder is indeed a series, which will take into account a future where the first buyer is no longer alive, but where second, third,... buyers can profit from the earnings. Other assets that in theory might provide earnings indefinitely in time are, for example, shares of stock, among others.

2. A second criticism is that one might argue that although a series is needed to compute the exact value of some asset, one can just use a finite number 
of terms of the series to compute a good enough approximation of the value, let's say an approximation with precision $2^{-n}$, i.e. one can compute some function $l: \mathbb{N} \rightarrow \mathbb{N}$ such that

$$
\left|\sum_{t=1}^{\infty} e(t) u(t)-\sum_{t=1}^{l(n)} e(t) u(t)\right|=\left|\sum_{t=l(n)+1}^{\infty} e(t) u(t)\right| \leq 2^{-n}
$$

This is the case for consols, which make fixed payments along time. But what about other assets with a more rich behavior, like shares of stock? How many years do we have to consider to compute the price down to the cent? 2? 5? 10? 20? What if the earnings start to grow exponentially after this set period? We would miss important behavior from a "fat tail" (fat tail myopia) and hence our approximation would not be as good as we thought it was. This is also the essence of our non-computability result, since Theorem 12 implies that, in general, no computable function $l$ satisfying (12) exists (although such function $l$ exists for some particular cases, like the example of consols).

3. A third criticism is simply the following remark: "But in practice there are people who compute the values of, say, shares of stock using the DCFM. How can this problem be non-computable?" Indeed, the DCFM is used to determine the fundamental value of a share of stock, as often advertised by investments banks as "target prices", even if in practice people tend to use simpler approaches ( $\mathrm{P} / \mathrm{E}$ ratios, etc. See [4]) as rude proxies for the fundamental value. However these values are only approximations, since the input data is obtained from estimates of the earnings for the next, say, 5 years and by using simple assumptions like those of the well-known Gordon's model, where earnings are supposed to grow after these 5 years at a constant rate with a fixed discount rate, cf. [4]. For instance, in this case, the price would be

$$
\left.\sum_{t=1}^{5} e(t) u(t)+\sum_{t=1}^{\infty} \frac{e(5)(1+g)^{t}}{(1+r)^{t}}\right)
$$

(after 5 years the earnings are assumed to grow at rate $g$, taking as base those earnings obtained in the 5 th year, $e(5))$. But using this approach we might fall again into fat tail myopia, since we are just replacing the tail for $t \geq 6$ by an arbitrary tail (in this case by the one given by Gordon's model). But it could happen (and it does happen in practice) that, after some time, we realize that some assumptions done about the future are wrong (the company is starting to grow much more quickly/slower than expected) and this information is updated in the stock price through a significant increase/decrease of its value. Thus we would not get a single stock price, but rather a time series in which the value will change accordingly to new and better information. Under the DCFM (thus in an ideal world removed from speculators), variations of stock prices are classically attributed to 
inherently uncertain input data, and as we get more reliable information, we revise the corresponding stock price. In this paper we suggest that uncertainty of input data might not explain all these price oscillations since, even if we do know precisely all the input data and this input data is finite, we might not be able to compute the fair price (or a good enough approximation) of the stock (of course, in general, the inputs are infinite in size. But in Lemma 11 we have seen that even if they are computable - and in this case they can be described in a finite manner through an algorithm - the result returned by the DCFM operator can be non-computable).

4. A fourth and final criticism is that one might claim that the input data used in the proof of Lemma 11 is not realistic. We cannot assert that it is fully realistic (this is more a theme for finance or economics), but we have tried to ensure that it is as close to reality as possible. For instance, there are assets with constant earnings along time as in the proof of Lemma 11 (e.g. bonds, consols). Relatively to the discount function, the simplest type of discount function, which assumes a fixed constant rate $r$, can be expressed as the power function

$$
u(n)=\frac{1}{(1+r)^{n}}
$$

which is not too dissimilar from the function

$$
u(n)=\frac{1}{2^{a(n)}}
$$

used in Lemma 11. It is true that the latter discount function has a more complicated behavior than that obtained by using a constant rate. But the constant rate discount function is certainly too simplistic. For example, the discount rate depends on inflation (one can always buy hard assets, e.g. gold, which will value accordingly to inflation, instead of putting money into a savings certificate as in Section 2), and inflation is not constant. We could perhaps bound this inflation within certain limits (the stated objective of several central banks), but even the variation within this band may be non-computable. Moreover, from time to time, there have been unexpected surges on inflation, which sometimes got unanticipated heights. An example is the German hyperinflation of the 1920s. In the year of 1923 alone, the inflation was over $10^{10 \%}$ [12, p. 39]. Similar phenomena has been observed more recently in other countries such as Nicaragua, Bosnia and Herzegovina, Ukraine, or Zimbabwe (for example, in November 2008, the month-to-month inflation rate in Zimbabwe was $79600000000 \%$ [13]). Political instability and civic unrest are leading causes for hyperinflation, which make this phenomenon quite difficult to predict. 


\section{Conclusion}

In this paper we have shown that the mathematical model used to price many assets like bonds, stocks, etc. is non-computable. Of course, mathematical models are idealizations of the reality. Nevertheless ours results introduce the intriguing idea that unpredictable behavior of financial markets may be partially due to inherent non-computability.

Acknowledgments. D.S. Graça was partially supported by Fundação para a Ciência e a Tecnologia and EU FEDER POCTI/POCI via SQIG - Instituto de Telecomunicações through the FCT project PEst-OE/EEI/LA0008/2011.

\section{References}

[1] A. M. Turing. On computable numbers, with an application to the Entscheidungsproblem. Proc. London Math. Soc., (Ser. 2-42):230-265, 1936.

[2] Y. Matijasevič, M. Davis, and H. Putman. Hilbert's Tenth Problem. The MIT Press, 1993.

[3] A. Damodaran. Investment Valuation: Tools and Techniques for Determining the Value of Any Asset. John Wiley \& Sons, 2nd edition, 2002.

[4] Z. Bodie, A. Kane, and A. Marcus. Investments. McGraw-Hill, 7th international edition, 2008.

[5] K. Weihrauch. Computable Analysis: an Introduction. Springer, 2000.

[6] K.-I Ko. Computational Complexity of Real Functions. Birkhäuser, 1991.

[7] M. B. Pour-El and J. I. Richards. Computability in Analysis and Physics. Springer, 1989.

[8] V. Brattka, P. Hertling, and K. Weihrauch. A tutorial on computable analysis. In S. B. Cooper, , B. Löwe, and A. Sorbi, editors, New Computational Paradigms: Changing Conceptions of What is Computable, pages 425-491. Springer, 2008.

[9] M. Sipser. Introduction to the Theory of Computation. Course Technology, 2nd edition, 2005.

[10] A. M. Turing. On computable numbers, with an application to the Entscheidungsproblem. a correction. Proc. London Math. Soc., (Ser. 2-43):544-546, 1937.

[11] P. Odifreddi. Classical Recursion Theory, volume 1. Elsevier, 1989.

[12] C. Bresciani-Turroni. The Economics of Inflation: A Study of Currency Depreciation in Post-War Germany. Routledge, 2003, first printed in 1937. 
[13] S. H. Hanke and A. K. F. Kwok. On the measurement of zimbabwe hyperinflation. The Cato Journal, 29(2):353-364, 2009. 